\title{
Research advances in molecular mechanisms underlying the pathogenesis of cystic fibrosis: From technical improvement to clinical applications (Review)
}

\author{
TAO WEI $^{1 *}$, HONGSHU SUI $^{1 *}$, YANPING SU $^{1 *}$, WANJING CHENG ${ }^{1}$, \\ YUNHUA LIU $^{1}$, ZILIN HE ${ }^{1}$, QINGCHAO JI ${ }^{1}$ and CHANGLONG XU ${ }^{2}$ \\ ${ }^{1}$ Department of Histology and Embryology, Shandong First Medical University and \\ Shandong Academy of Medical Sciences, Tai'an, Shandong 271000; ${ }^{2}$ Reproductive Medical Center, \\ Nanning Second People's Hospital, Nanning, Guangxi Zhuang Autonomous Region 530031, P.R. China
}

Received April 29, 2020; Accepted September 17, 2020

DOI: $10.3892 / \mathrm{mmr} .2020 .11607$

\begin{abstract}
Cystic fibrosis (CF) is a chronic disease causing severe impairment to the respiratory system and digestive tracts. Currently, $\mathrm{CF}$ is incurable. As an autosomal recessive disorder, the morbidity of CF is significantly higher among Caucasians of European descent, whereas it is less pervasive among African and Asian populations. The disease is caused by identical mutations (homozygosity) or different mutations (heterozygosity) of an autosomal recessive mutation at position 7q31.2-q31.1 of chromosome 7. Diagnostic criteria and guidelines work concurrently with laboratory detection to facilitate precise CF detection. With technological advances, the understanding of $\mathrm{CF}$ pathogenesis has reached an unprecedented level, allowing for increasingly precise carrier screening, more effective early stage CF intervention and improved prognostic outcomes. These advances significantly increase the life quality and expectancy of patients with CF. Given the numerous improvements in the field of $\mathrm{CF}$, the current review summarized the technical advances in the study of the molecular mechanisms underlying CF, as well as how these improvements facilitate the clinical outcomes of CF. Furthermore, challenges and obstacles to overcome are discussed.
\end{abstract}

Correspondence to: Dr Changlong Xu, Reproductive Medical Center, Nanning Second People's Hospital, 13 Dancun Road, Nanning, Guangxi Zhuang Autonomous Region 530031, P.R. China E-mail: xuchanglong2011@hotmail.com; xc12019paper@163.com

*Contributed equally

Abbreviations: CF, cystic fibrosis; CFTR, cystic fibrosis transmembrane conductance regulator

Key words: cystic fibrosis, cystic fibrosis transmembrane conductance regulator, cystic fibrosis transmembrane conductance regulator mutation, sweat test, gene therapy

\section{Contents}

1. Introduction

2. Molecular mechanism underlying the CFTR mutation in $\mathrm{CF}$

3. Technical advances and implementations in $\mathrm{CF}$

4. Molecular regulators in $\mathrm{CF}$

5. Clinical applications of $\mathrm{CF}$-associated molecules

6. Challenges and perspectives

7. Conclusions

\section{Introduction}

Cystic fibrosis (CF) is an autosomal recessive disease that can be attributed to the disrupted function of the CF transmembrane conductance regulator $(C F T R)$ gene $(1,2)$. Although $\mathrm{CF}$ predominantly affects the lungs, it is a multiorgan disease (3), affecting the pancreas, liver, kidneys (4) and intestine (5). CFTR mutation is the cause of the pathogenesis of CF (6) and CF is generally a result of the deletion of the phenylalanine at the 508th position of CFTR, which is induced by the loss of three nucleotides (7). In vertebrates, CFTR serves as a membrane protein and participates in the functions of $\mathrm{Cl}^{-}$channels $(8,9)$. Due to its important regulatory functions, CFTR is ubiquitous throughout the body and is expressed in epithelial cells in the kidney, pancreas, airway, intestine (4), sweat glands and the male reproductive tract, where it serves a fundamental role in the transepithelial fluid (10). The number of identified CF-associated mutations are increasing, with $\sim 1,700$ CFTR mutations being previously recognized to be CF-prone (11); however, this number was re-estimated at 383 in 2019 according to the Clinical and Functional Translation of CFTR website (www.cftr2.org; date of access: 05/08/20). These potential mutations were screened by a specific criteria that determines the mutations responsible for the onset of $\mathrm{CF}$ : Firstly, the mutation could cause changes in the amino acid sequence, affecting both the expression level and functions of CFTR (12); secondly, the mutation introduces premature signals and exhibits a novel amino acid sequence that is absent in the normal CFTR gene (13). 
The prevalence of CF varies with ethnicity $(14,15)$. The relatively high incidence of $\mathrm{CF}$ among Caucasians may be attributed to their increased number $(>1,400)$ of CFTR mutations (16). Furthermore, while 1/3,000 of Caucasians will develop CF, the incidence is lowered to $1 / 15,000$ among the African population, further decreasing in Asian populations to $1 / 30,000$, compared to the aforementioned two ethnic groups (15). The ratio of CF incidence between male and female is 1:1; however, the mortality rate of CF-associated lung infections is higher among female patients as they are subjected to greater deterioration of pulmonary function at puberty. These gender/age gaps have been proposed to be a result of the elevation in the hormone secretion (including estrogen) in adults, which may disrupt airway ion transport in lungs (17).

There are two major molecular subtypes of CF: Classic CF and non-classic $\mathrm{CF}$. Non-classic $\mathrm{CF}$ refers to $\mathrm{CF}$ with better prognostic outcome, as certain functions of the CFTR protein are preserved, providing advantages for survival. Non-classic patients with CF have $\geq 1$ copy of a defect CFTR gene with partially conserved CFTR protein functions. Due to the partial preservation of pancreatic exocrine functions, the symptoms of digestion disorders are less common among patients suffering from this milder type of CF. In contrast, patients suffering from classic CF have completely lost their functional CFTR protein. This subtype is characterized by persistent bacterial infection in the airways and sinuses, disrupted fat digestion due to the lack of pancreatic exocrine, male dysgenesis due to obstructive azoospermi and increased sweat $\mathrm{Cl}^{-}$levels (18-20).

The original description of CF can be dated back to 1938 (21). Since then, progress in the understanding of CF has been made in a step-by-step manner and the following 50 years has witnessed remarkable improvement in life expectancy and life quality among patients with CF (22), which may be attributed to technological innovations. In the late 1950s, a stimulated sweat test to diagnose patients with $\mathrm{CF}$ through $\mathrm{Cl}^{-}$or Na levels was developed (23) based on the recognition of the altered electrolyte composition in sweat (24). The preliminary works contributed markedly to the diagnosis of $\mathrm{CF}$ and the understanding of $\mathrm{CF}$ was further promoted nearly 30 years later due to the discovery of the CFTR gene, a key mediator of CF (9), which enabled the diagnosis of CF by directly identifying 2 mutated CF alleles (25). Aside from improved diagnostics, numerous therapies have been applied to treat $\mathrm{CF}$, including antibiotics against infections, nutritional supplementation and/or lung transplantation, through which the life expectancy of patients with CF can be significantly prolonged (26).

Despite prognostic improvements of $\mathrm{CF}$, the median survival of patients with CF is $<50$ years (22). As described above, as the molecular mechanisms in CF are associated with ethnical and sex differences in terms of incidence rate, they can also be used to determine phenotypes of CF. Therefore, the innovation of methods for the detection and identification of $\mathrm{CF}$ at the molecular level will be beneficial to the diagnosis and prognosis of $\mathrm{CF}$. The current review aimed to summarize the recent research advances of $\mathrm{CF}$, including technical improvement in the understanding of the molecular mechanism of CF. Additionally, the increasing number of molecular markers that have the potential to improve diagnostic and prognostic outcomes of $\mathrm{CF}$ are discussed. Briefly, a PubMed (pubmed.ncbi.nlm.nih.gov; date of access: 13/08/2020) search was conducted using the following key words: 'Cystic fibrosis', 'molecular', 'diagnosis', 'prognosis' and 'therapy'. Examples were chosen as long as they fulfilled one of the following eligibility criteria: i) Provided genetic information regarding the pivotal role of CFTR in CF; ii) described the latest progresses in parsing the molecular mechanisms underlying $\mathrm{CF}$ using novel techniques; iii) demonstrated the association between $\mathrm{CF}$ and other bioactive molecule (molecular chaperone) and the potential clinical implementations, including CF diagnosis and treatment.

\section{Molecular mechanism underlying the CFTR mutation in $\mathbf{C F}$}

Molecular structure of CFTR. The molecular weight and length of the CFTR protein are 1,480 amino acids and $168,173 \mathrm{Da}$, respectively $(7,12,27)$. The length of its coding sequence, which encodes the amino acid sequence for protein products, is $4,443 \mathrm{bp}$ (28). The intron-free sequence of the CFTR transcript is $6,129 \mathrm{bp}$ in length $(12,28)$, whereas the normal allelic variant for CFTR is $\sim 250,000$ bp in length and contains 27 exons $(12,28)$. CFTR is comprised of 5 functional domains (12): Two domains (MSD1 and MSD2) controlling membrane-spanning, which constitute the ion channel for $\mathrm{Cl}^{-}$transportation; an $\mathrm{R}$ domain, which exerts regulatory roles; and two domains (NBD1 and NBD2) that bind and catalyze the hydrolysis of adenosine triphosphate.

Biological functions of CFTR. The CFTR protein is positioned in the cell membrane (29) and is associated with proteins involved in the active transportation of material through the cell membrane $(12,30)$. Specifically, CFTR regulates the movement of $\mathrm{Cl}^{-}$. Therefore, defects in CFTR gene can render the CFTR protein absent or dysfunctional, thereby blocking the transportation of $\mathrm{Cl}^{-}$to the cell surface $(29,30)$. Additionally, aside from $\mathrm{Cl}^{-}$, CFTR regulates the epithelial Na channel (31). Abnormalities in the CFTR protein disrupt the balance between $\mathrm{Na}$ and $\mathrm{Cl}^{-}$ions $(30,32)$, which leads to changes in mucous constituents and abnormal reabsorption of $\mathrm{H}_{2} \mathrm{O}$. This produces a layer of thick, sticky mucus that cannot be removed by cilia, which eventually causes inadequate mucociliary function and chronic infections (33). This can be fatal. In the lungs, accumulated mucus can become infested with bacteria and the chronic inflammation leads to pneumonia, resulting in deterioration with life-threatening difficulties in breathing. Given the molecular mechanisms of the deficiency of CFTR, the common symptoms of CF include severe cough and shortness of breath; however, CF can also lead to abnormal bowel movements, difficulty in gaining weight and infertility $(12,29)$.

Classification of CFTR mutations. Based on the effects on protein translation, cellular processing or channel gating of CFTR $(28,30)$, several different classification systems (Fig. 1) have been proposed over the years. Generally, the Class 1 mutation results in severe disease, as this mutation prevents the CFTR protein from being generated. Patients with Class 1A mutation do not synthesize any CFTR mRNA. Furthermore, patients with Class 1B produce damaged CFTR mRNA, 


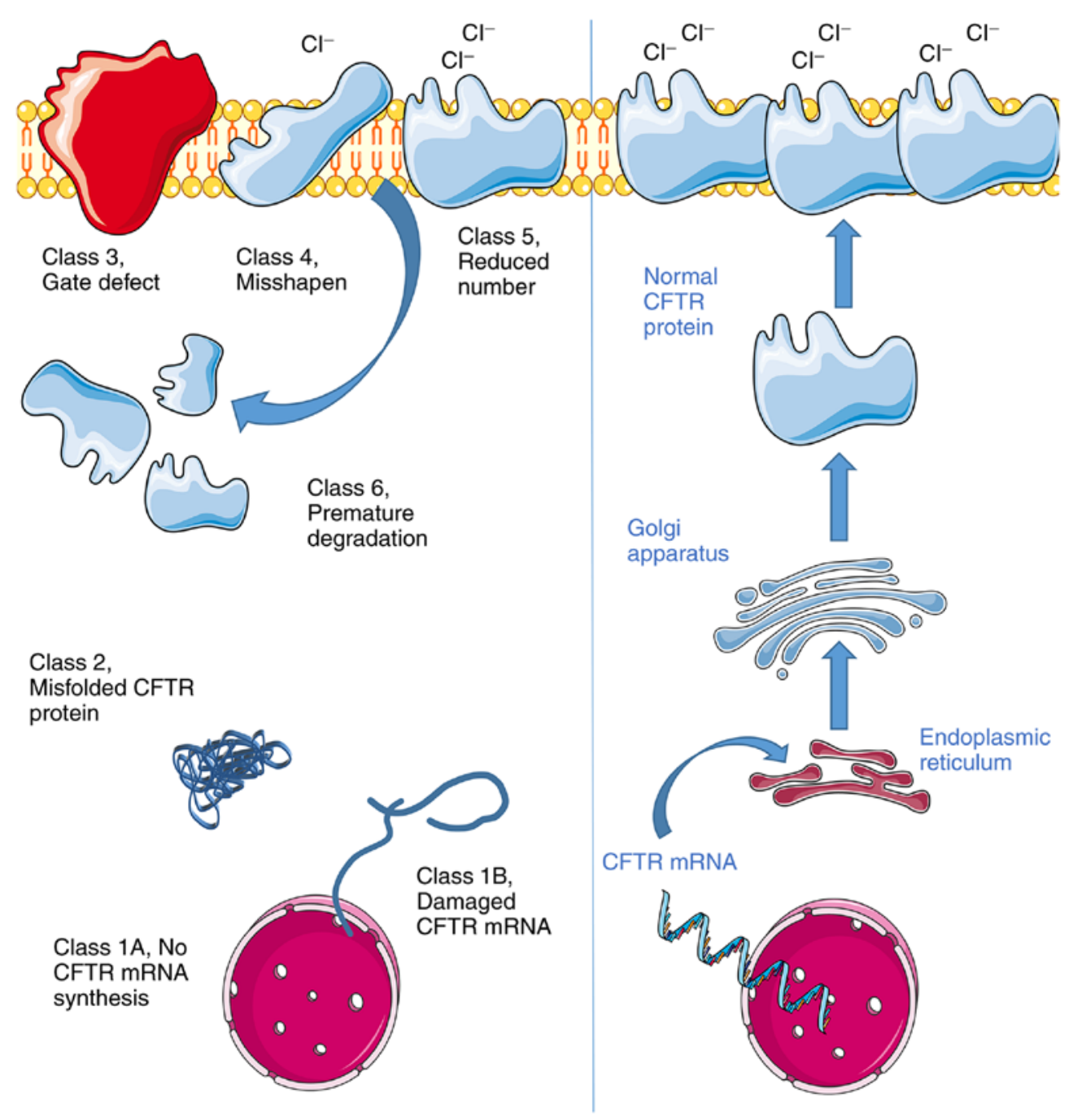

Figure 1. Different types of CFTR mutations. Generally, intact CFTR mRNA can be generated from the cell nucleus and following correct folding, sufficient amount of normal CFTR protein is transported to the cell membrane to serve as a $\mathrm{Cl}^{-}$channel. In contrast, different malfunctions in this multi-step process lead to different CFTR defects. CFTR, cystic fibrosis transmembrane conductance regulator; $\mathrm{Cl}^{-}$, chloride ion.

which cannot be converted into protein (28). In Class 2 mutations, the CFTR protein is produced; however, it is misfolded. The misfolded protein will be prevented from migrating to the cell membrane. In Class 3 mutations, channels in the CFTR protein are not properly opened due to gate defect $(29,32,34)$. For the Class 4 mutation, while the CFTR protein is responsive to cell signaling, it is misshapen, resulting in a limited flow of $\mathrm{Cl}^{-}$ions. Furthermore, in Class 5 mutations, insufficient CFTR protein is produced, leading to a reduction in the number of CFTR protein channels at the cell membrane (35). In class 6 mutations, less stable protein is prematurely degraded after it reaches the cell surface. Relatively, this mutation is less severe compared with the other mutations and, therefore, is a milder subtype. Generally, the Class 1, 2 and 3 mutations are more common and responsible for insufficiency in the organs suffering from $\mathrm{CF}$.

\section{Technical advances and implementations in CF}

PCR analysis of $C F$. PCR is a widely used laboratory technique that allows for the semi-quantification of mRNAs. As early as 1992, allelic specific-PCR was used for detecting
F508del mutation in CFTR (36). In the following decades, expression of CFTR had been verified by various models by reverse transcription-quantitative PCR (RT-qPCR). Certain implementations of RT-qPCR in CF include the following: i) In CF cell IB3-1 transduced with CFTR vectors, CFTR mRNA expression was detected using RT-qPCR, whereby the efficiency of transduction was measured (37); and ii) multiplex fluorescent RT-qPCR was used for scanning the exons to detect large CFTR rearrangements (38).

Aside from the aforementioned utilizations in CFTR detection, PCR is frequently performed to examine bacterial infection in $\mathrm{CF}$; for instance, PCR was used to detect Aspergillus fumigatus DNA, which commonly infects the airways of patients with CF (39), in samples collected from patients with CF. Preimplantation genetic testing (PGT) is an important method to detect CF before or at pre-embryonic stages (40). The updated version of the PGT guidelines regarding $\mathrm{CF}$ proposed that $\mathrm{PCR}$ analysis should be performed to detect the causative mutation(s), along with associated polymorphisms within or near to the CFTR gene (41). Nevertheless, PCR has its own limitations. For instance, during unequal allelic PCR amplification, allele dropout can hamper the 
detection of CFTR mutations, as the annealing of a primer to the matched allelic sequence is predominated as compared to its mismatched counterpart (42).

Implementation of next-generation sequencing (NGS) in $C F$. The revolutionary innovation of sequencing technologies, including NGS platforms, allows for the detection of a broader spectrum of potential mutations in $\mathrm{CF}$, particularly single-nucleotide polymorphism (43), which are hypothesized to be a cause of CF (44). NGS outperformed whole genome sequencing in terms of cost-efficiency and its accuracy is guaranteed by stringent thresholds during data processing (43). Therefore, NGS has been widely used in carrier (at-risk individual) screening, including CFTR mutation screening, to improve genetic counseling and reduce the incidence of $\mathrm{CF}$ among carriers' (at-risk-couples) descendants (43). In another study of methodology establishment, NGS-based expanded carrier screening, which determines variants through hybridization capture gene enrichment, identified several genetic alterations, including copy-number variants in the CFTR gene. The combination of NGS and variant interpretation achieved higher accuracy in identifying $\mathrm{CF}$-associated phenotypes compared with the traditional method (23 variants screening) (45). Furthermore, by retrospectively performing NGS assays on patients with single CF mutated screened by sweat tests, all CFTR mutations were correctly detected, indicating that NGS assays were completely concordant with traditional methods (46). These reports demonstrated the effective implementation of NGS in CF detection, particularly at the early stage of the disease.

Gene editing for CF. Clustered regularly interspaced short palindromic repeats (CRISPR)/CRISPR-associated protein 9 (Cas9) is an emerging technology by which Cas9 proteins work in conjunction with guide RNA molecules and locate the site of target DNA sequence prior to cutting it out (47), following which the gap can be filled with the corrected gene sequence through the endogenous cellular regeneration. Therefore, this technology can be implemented in different single-gene-driven heritable deficiencies. With the advances in such gene editing technology, a promising future has been demonstrated in regard to the replacement of defective CFTR gene at the DNA level, through which normal CFTR function could be fundamentally restored (48). Although gene editing is still at its infant stage due to the relatively high off-target rate (49), it has demonstrated greater potential when compared to traditional CF therapies targeting (instead of editing) DNA, RNA or proteins. For instance, the functional repair of CFTR has been successfully performed in an in vitro model derived from stem cells of patients with $\mathrm{CF}$, namely intestinal organoids (50). Another approach, Zinc finger nucleases-mediated gene editing, was used to correct defective CFTR in induced pluripotent stem cells (51). Notably, a mutation which could cause $\beta$ thalassemia was corrected in human embryos using CRISPR/Cas9, indicating the capability of embryonic gene editing with this technique (52). Thus, we hypothesized that CRISPR/Cas9-induced gene editing in embryos threatened with potential mutations is a promising for the future treatment of CF.

\section{Molecular regulators in $\mathrm{CF}$}

Non-coding RNAs (ncRNAs) in CF. Although CF is monogenic, the phenotypes of patients with $\mathrm{CF}$ are heterogeneous, which may be attributed to multiple regulators that contribute to $\mathrm{CF}$ pathogenesis (53). Non-coding (nc)RNAs are a type of RNA molecule that do not translate into a protein. Instead, ncRNAs exerts regulatory roles in multiple biological processes, such as translation (54), RNA splicing (55) and gene regulation (56), among which microRNA (miRNA or miR), long non-coding RNA (lncRNA) and circular RNA (circRNA) have been extensively studied. The current review discusses several ncRNAs that participate in $\mathrm{CF}$ pathogenesis.

miRNA and CF. Previous studies have demonstrated that miR-145, miR-494 and miR-101 directly or indirectly target and regulate CFTR $(53,57-59)$. The inhibition of miR-145 through peptide nucleic acids was reported to promote the expression of CFTR, as miR-145 binds to the 3'-untranslated region (3'UTR) of the CFTR gene (57). Additionally, the interaction of miR-494/miR-101 and CFTR was verified and confirmed through luciferase reporter assays (58). Considering evidence that has demonstrated the inhibitory effects of these miRNAs on CFTR, the exacerbated pulmonary condition caused by air pollution or cigarette smoke was studied and attributed to elevated miR-101 and miR-144 (60). Although most miRNAs that directly target CFTR serve as suppressors, certain miRNAs exert indirect regulatory roles on CFTR to promote its expression (Fig. 2). For instance, in primary epithelial cells derived from CF bronchia (CFTR defect phenotype), miR-138 was reported to downregulate the expression of SIN3 transcription regulator family member A (SIN3A), a negative transcriptional regulator of CFTR (61). In an indirect manner, miR-138 promoted the expression of CFTR through alleviating the repression that SIN3A imposed on CFTR.

Furthermore, miRNAs participate in other biological processes. In CF lung epithelial cells, miR-155 promoted inflammation through the inositol 5'-phosphatase 1-PI3K/Akt cascade. Moreover, as chronic bacterial lung infection is a major cause of CF morbidity, exhaled breath condensate was used for miRNA profile analysis of patients with $\mathrm{CF}$ with microbial infection. The results demonstrated that 6 miRNAs (has-miRNA-432-5p, hsa-miRNA-3170, hsa-miR449c, hsa-miR-1276, hsa-miR-1247 and hsa-miR-548) were identified as potential biomarkers for patients with $\mathrm{CF}$ and chronic Pseudomonas infection (62). These reports indicated that miRNAs serve crucial functions in the pathogenesis of $\mathrm{CF}$ and are key diagnosis and prognosis markers for $\mathrm{CF}$.

lncRNA and CF. Dysregulation of IncRNAs have been reported to be associated with chronic pulmonary infection, adaptive immune responses and inflammation in patients with CF (63). By working concurrently with several proteins, lncRNA BGas (a novel long noncoding RNA located in the intron 11 of the CFTR gene) regulated CFTR by regulating its local chromatin and DNA structure (53). Microarray profiling of lncRNAs revealed one upregulated (X-inactive specific transcript) and several downregulated (HOX Transcript Antisense RNA, Metastasis Associated Lung Adenocarcinoma Transcript 1 and Toll Like Receptor 8 Antisense RNA 1) lncRNAs in 


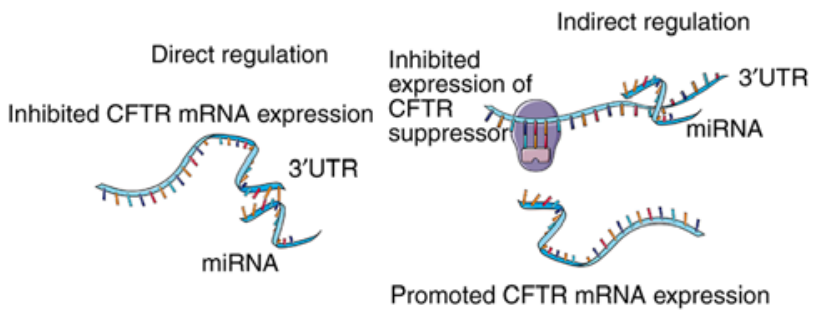

Figure 2. miRNA regulation of CFTR. The majority of studies have focused on the direction of miRNAs in the regulation of CFTR, whereby miRNAs lead to silence or degradation of CFTR mRNA by binding to its 3'UTR. Additionally, miRNAs inhibit the expression of certain CFTR suppressors, through which they promote the expression of CFTR mRNA. miRNA, microRNA; CFTR, cystic fibrosis transmembrane conductance regulator; UTR, untranslated region.

the patients with CF compared with the matched non-CF controls (64). Although the current evidence regarding the association between lncRNAs and CF is relatively limited, it is notable that in a comparative study, 636 and 1,974 differentially expressed lncRNAs were found in two groups of patients suffering from CF airway epithelium or CF lung parenchyma (compared with the matched non-CF counterparts), respectively. By analyzing these lncRNAs in a comprehensive manner, the antisense lncRNA RN7SK Pseudogene 237 was found to be significantly altered in CF airway tissues, whereas the downregulation of two intergenic lncRNAs, long intergenic non-protein coding RNA (LINC)01023 and LINC00176 were confirmed in CF parenchyma tissues (65). Additionally, in silico analysis of RNA-seq data demonstrated that Pseudomonas aeruginosa infections lead to 108 altered lncRNAs expression between respiratory epithelial cells derived from patients with $\mathrm{CF}$ and non-CF donors. Among these lncRNAs, LINC00862 and CTD-2619J13 were significantly altered at different time points throughout the process $(0 \mathrm{~h}$ prior to infection and 2,4 and $6 \mathrm{~h}$ following infection) (63). These studies indicated that lncRNAs exerted important regulatory roles in $\mathrm{CF}$, which still remain to be fully elucidated.

circRNA and $C F$. In the field of RNA, circRNAs are endogenous ncRNAs. These RNAs have been identified in organisms, including eukaryotes, archaea, bacteria and viruses, and act as a sponge for certain miRNAs in pulmonary diseases (66). Currently, reports regarding the potential roles of circRNAs in $\mathrm{CF}$ are relatively limited. However, due to their close regulatory association with miRNAs, it is likely that circRNAs participate in the progression of CF. Specifically, in bladder cancer, circ-solute carrier family 8 member A1 was reported to be a sponge of miR-494 (67), whereas direct interactions between circ-baculoviral IAP repeat containing 6 and miR-145 were indicated in embryonic stem cells (68). Reports concerning miR-494 and miR-145 and their involvement in CFTR were revealed $(57,58)$, indicating that these regulatory circRNAs may regulate CFTR through these miRNAs. Analogously, it has been hypothesized that numerous miRNAs regulating CFTR may be targets for various circRNAs, which might be involved in $\mathrm{CF}$. This is a novel research avenue to consider in the future since the elucidation of the mechanisms driven by cirRNAs will provide a further understanding on the pathogenesis of $\mathrm{CF}$ and its complications.
Epigenetic modifications in $C F$. Epigenetics is a mechanism that alters gene expression without changing the fundamental DNA sequence. The two mostly known mechanisms underlying epigenetic modifications include histone modifications and DNA methylation (69), both of which are involved in regulation of CFTR. It had been hypothesized that aside from ncRNAs, epigenetics is a contributing factor in the disease variability of CF (70). Additionally, epigenetic mechanisms have been proposed to be an activator of host defenses that induce a robust immune response (71). The association between immunity and epigenetics has demonstrated that DNA methylation at numerous gene loci in lung macrophages was responsible for the malfunction of innate immune cells in lungs with CF (72). Additionally, differentially altered DNA methylation at $\mathrm{CpG}$ sites was associated with lung function and their overexpression was demonstrated in numerous regulatory genes responsible for cell adhesion (for example, ETS homologous factor) and inflammatory responses (for example, baculoviral IAP repeat-containing protein 1) (73) in nasal epithelial samples from patients with CF (74). Furthermore, acetylation has been proposed to be associated with CF. For instance, the inhibition of histone deacetylase (HDAC)7 was demonstrated to restore the function of F508del (75) and HDAC2 was reported to be responsive to defective CFTR function (76). A previous study demonstrated that microtubule deacetylase regulated cholesterol accumulation and NF- $\mathrm{NB}$ activation in $\mathrm{CF}$ cells through the HDAC6-Ac-tub cascade, which corroborated with the findings that HDAC6 may be a therapeutic site for various $\mathrm{CF}$ phenotypes (77). Collectively, therapeutic approaches for $\mathrm{CF}$ that target epigenetic mechanisms have been considered promising, as epigenetic alterations are dynamic and reversible. However, epigenetic therapy of CF disease is still at its infant stage (78).

\section{Clinical applications of CF-associated molecules}

Molecular diagnosis of $C F$. The association between clinical presentations and residual CFTR function has been established (79). Congenital absence of the vas deferens is established when the proportion of normal CFTR function is $<10 \%$, as this number decreases $(<5 \%)$, positive sweat test results could be supported and patients might suffer from pulmonary infection when CFTR function further drops to $<4.5 \%$. The worst cases (CFTR function $<1 \%$ ) lead to pancreatic insufficiency, aside from the aforementioned symptoms (80). As varying molecular subtypes are associated with different phenotypes, experts from the 'Cystic Fibrosis Foundation' convened a panel of criteria for diagnosing CF in 1996 (81,82). Several tests, including the sweat test $(83,84)$, nasal potential difference (NPD) (85-87), DNA screening $(88,89)$ and a ciliary test, were recommended. The current review discussed traditional (regular) approaches (sweat test and NPD measurement) and novel methods.

The sweat test is an effective method for detecting $\mathrm{CF}$, covering all age ranges $(83,84)$. However, the application of creams and lotions within 1 day prior to sweat collection can disrupt the precision of diagnosis. The criteria for determining CF varies based on different ages. In infants up to 6 months of age, a CF diagnosis is very unlikely if the level of $\mathrm{Cl}^{-}$is not $>29 \mathrm{mmol} / \mathrm{l}$. However, the possibility of establishing a 
CF diagnosis increases when $\mathrm{Cl}^{-}$levels range 30-59 mmol/1. Generally, the diagnosis of CF can be confirmed when this level is $>60 \mathrm{mmol} / \mathrm{l}$. The criteria vary slightly in patients aged $>6$ months. $\mathrm{CF}$ cannot be diagnosed when the $\mathrm{Cl}^{-}$concentration is $<39 \mathrm{mmol} / \mathrm{l}$. When levels range $40-59 \mathrm{mmol} / \mathrm{l}$, a higher probability of $\mathrm{CF}$ is expected. The diagnosis of $\mathrm{CF}$ can be established if the $\mathrm{Cl}^{-}$levels are $>60 \mathrm{mmol} / \mathrm{l}$. Collection of a sufficient volume of sweat is required for laboratory assays, through which $\mathrm{Na}$ and $\mathrm{Cl}^{-}$concentrations are determined. Incorrect results occur due to contamination, evaporation, insufficient sample and technical errors $(83,84)$.

NPD measurement is used to follow-up patients with CF (85-87). NPD is generally used to evaluate the voltage between the reference electrode and the exploring electrode, which is sensitive and specific (85-87). In vivo, NPD provides data about incorrect ion transport due to CFTR protein dysfunction in the nasal epithelial cells of patients. Ancillary test is used to verify the phenotype of patients and identify ion channel abnormality. However, specific skills are required to perform the test and interpret the results.

DNA screening can detect severe mutations, including F508del and minor mutations such as the $5 \mathrm{~T}$ variant, and is particularly useful to detect $\mathrm{CF}$ in patients who are unable to perform the sweat test $(88,89)$. This method can provide a general idea associated with the severity of the illness and can detect less severe CF variants, including azoospermia and congenital bilateral absence of the vas deferens (90). Previously, children suffering from CFTR-associated metabolic disorders were classified into non-typical or moderate type of $\mathrm{CF}$. However, these indistinct categories lack stringent criteria. Therefore, this resulted in ambiguities in subtype stratification. Currently, DNA screening is widely used in newborn screening for improved stratification of the different subtypes of CF. Nevertheless, regular evaluation remains important (42). The most significant benefit of newborn screening and early diagnosis of $\mathrm{CF}$ is the possibility to treat disease-prone patients prior to the occurrence of serious symptoms (91).

One of the consequences of developing $\mathrm{CF}$ is the chronic pulmonary infection caused by colonized bacteria at an early age. Phenotypic features associated with $\mathrm{CF}$ diagnosis provides information about chronic sinopulmonary disease manifestation due to many microorganism, including Staphylococcus aureus, nontypeable Haemophilus influenzae, mucoid/non-mucoid Stenotrophomonas maltophilia, Pseudomonas aeruginosa and Burkholderia cepacian (92). These pathogenic bacteria can provoke gastrointestinal dysfunction responsible for intestinal, pancreatic, hepatic and nutritional troubles. Identification of the microorganism in patients with CF guides the path for subsequent antibiotic therapy (93). As mentioned in previous sections, microorganism detection can be performed by analyzing the expression profile of miRNAs (62) or other novel biomarkers (63). Therefore, traditional PCR analysis, microarray methods and NGS are capable of biomarkers profiling.

Molecular therapy for $C F$. Molecular therapy serves a crucial role in $\mathrm{CF}$ treatment. The current review discussed several alternatives, which are summarized in Fig. 3. In 1993, a gene therapy clinical trial was performed. The first trial focused on the nasal epithelium and adenovirus vectors containing the CFTR gene was used in an attempt to restore CFTR function (1,94-97). The rationale behind this method was to restore the dysfunctional gene or to supplement the patient with the corrected version of the protein prior to irreversible damage $(95,98,99)$. For this technique, the DNA has to penetrate the nucleus to be transcribed, which is the major barrier in gene therapy. In practice, gene therapy entails inhalation of a spray which delivers therapeutic DNA to the lungs. During the therapeutic process, either viral vectors (including adenovirus, lentivirus and herpes virus) or non-viral vectors (such as plasmids) were used. The best therapeutic outcome would be the successful replacement of the defective gene in the lungs to cure CF fundamentally. In other outcomes, CF symptoms are alleviated by decelerated disease progression; specifically, to clear aberrant and excessive secretions, combat pulmonary infections and to prevent intestinal obstruction (99). Additionally, gene therapy is the first and most advanced vector system using recombinant retroviruses ex vivo. In vivo gene therapy uses vectors based on the recombinant form of adenovirus. The recent virus-based system is an adeno-associated system and numerous vector systems have been validated in clinical trials involving human participants. Among them, adenoviruses and adeno-related viruses have been widely used $(37,100)$. Aside from virus vectors, cationic lipids-based vectors are also popular (99).

Transcript supplementation therapy using the correct version of CFTR mRNA transfected or transduced into the respective target cells has been documented (95). In this therapy, mRNA is actively producing CFTR in the cytoplasm, thereby circumventing the nuclear membrane. However, protein delivery is often ineffective and it is difficult to include natural posttranscriptional protein modifications. Additionally, RNA antisense therapy is taken into account in CF treatment. The hypothesis is to use inhaled RNA antisense to produce functional CFTR protein by inducing RNA to work more efficiently (53). Notably, nanotechnology using package miRNAs to treat $\mathrm{CF}$ was proven to be safe and effective. However, more research is required before applying this model to other diseases (66).

Another alternative for CFTR treatment includes modulator therapies (101), which can be categorized into two groups: Potentiators and correctors. The potentiators act on the CFTR ion channels. Therefore, these modulators are geared toward the class III subject group (gate defect), among which ivacaftor prolonged the opening of the CFTR channel, thereby facilitating $\mathrm{Cl}^{-}$ion flow (102). In January 2012, the U.S. Food and Drug Association approved ivacaftor use and, currently, ivacaftor is the only licensed CFTR potentiator (103). Observational data based on clinical and in vitro studies have indicated that ivacaftor is efficient for several mutations within classes III, IV and V in rat thyroid cell lines (102-104).

The correctors serve a key role in the transportation of nascent proteins (104). For instance, corrector lumacaftor is considered a stabilizer that increases the stability of mutated CFTR proteins, through which these proteins could be transported to the cell membrane more effectively and remain there for an extended period of time (105). These stable substrates could be further enhanced by potentiators (106). Monotherapy with lumacaftor, as a corrector, failed to demonstrate significant results in homozygous patients $(106,107)$. Furthermore, another type of corrector, tezacaftor, demonstrated great 


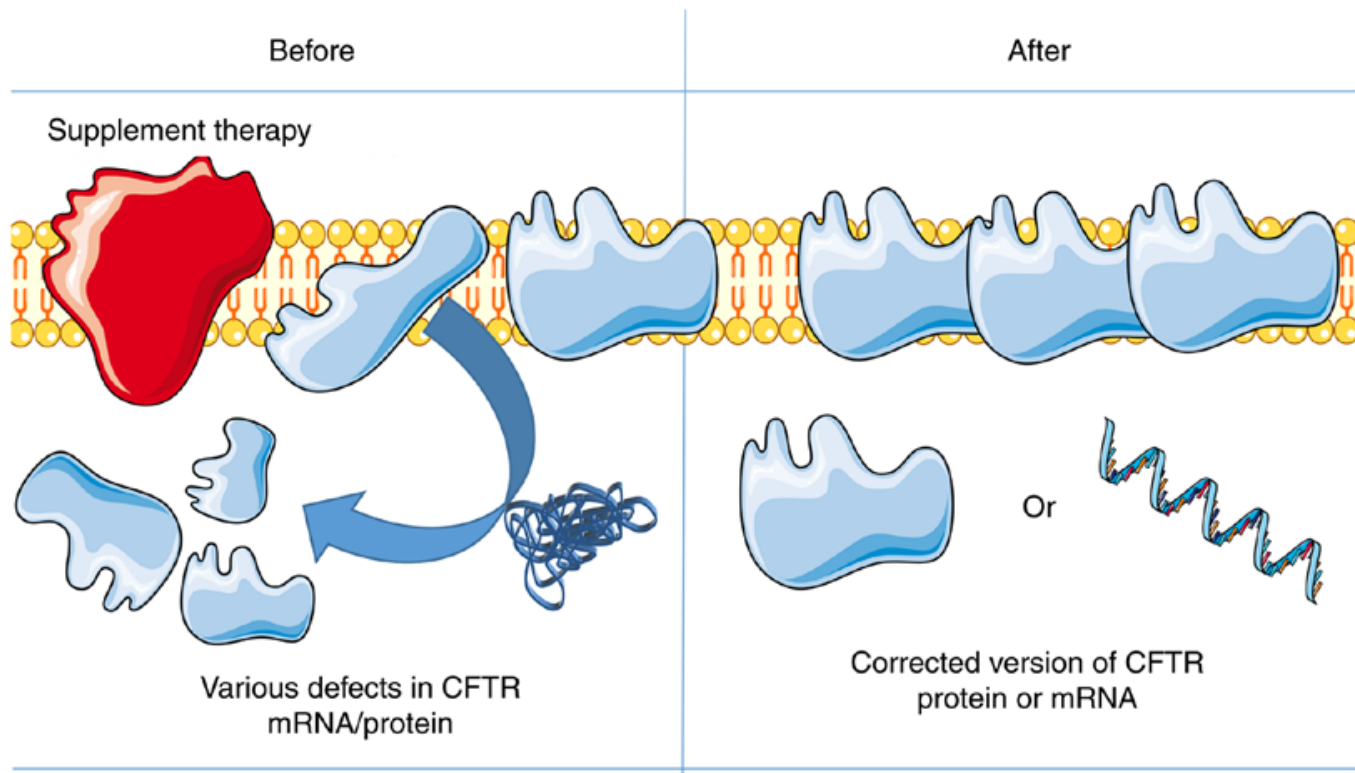

Gene therapy

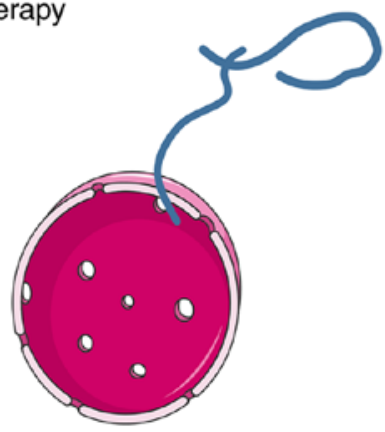

No CFTR mRNA production/damaged CFTR mRNA production

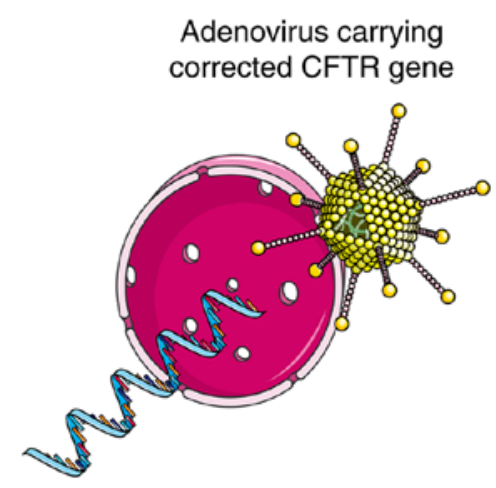

Recovered CFTR gene functions

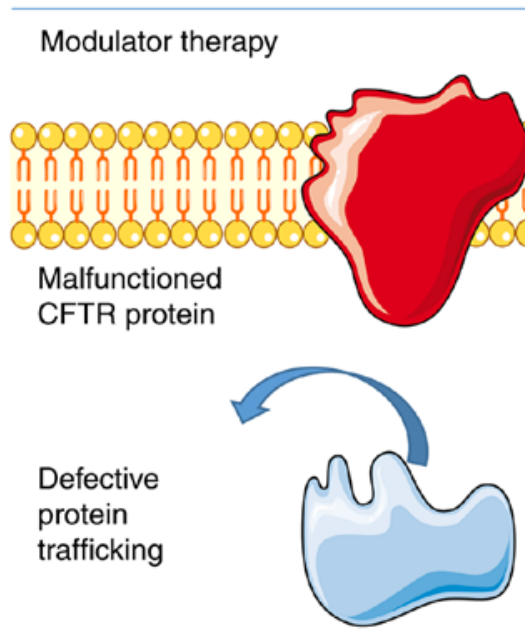

Insufficient immature CFTR protein

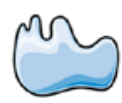

Amplifier

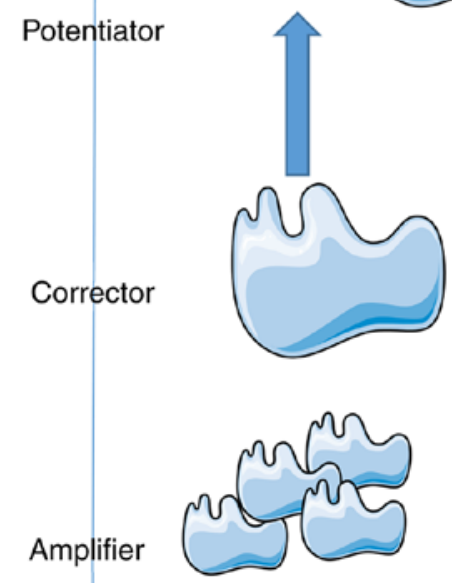

Reactivated

CFTR protein

Corrected

protein

trafficking

Increased

immature

CFTR protein

Figure 3. Schematic of several alternatives of molecular therapy. Comparisons were made between prior to and post-treatment. In supplement therapy, the corrected version of the CFTR protein (protein supplementation) or mRNA (RNA antisense therapy) were delivered to the cell. These exogenous molecules exerted their regulatory roles mainly in the cytoplasm. In gene therapy, packaged lentiviruses correcting the CFTR gene are directly inserted into the cell nucleus, thereby facilitating the normal transcription of CFTR mRNA. Furthermore, in modulator therapy, potentiators enhance the gating properties of malfunctioned CFTR, correctors induce correct CFTR protein folding/trafficking and amplifiers increase the production of immature CFTR protein, providing sufficient substrate for the corrector and potentiator. CFTR, cystic fibrosis transmembrane conductance regulator. 
result, improving the processing and trafficking of mutated CFTR, and promoting chloride transportation in bronchial epithelial cells derived from F508del/F508del donors, which were achieved without the problems associated with lumacaftor (for example, pulmonary exacerbation and increment in weight) (108). The underlying mechanism and propriety of tezacaftor are very close to those of lumacaftor (107), as tezacaftor therapy increases $\mathrm{Cl}^{-}$transport. When combined with ivacaftor, tezacaftor is efficient in transporting the CFTR protein to its correct position on cell surfaces (109-111). Therefore, potentiators and correctors are often combined to treat patients with CF. Specifically, CFTR potentiators increase the activity of CFTR on epithelial surfaces, whereas CFTR correctors promote processing and trafficking of mutated protein. In order to restore the availability and functionality of CFTR protein in the epithelium, CFTR modulator drugs are taken orally (106).

Furthermore, the third type of modulator, which is still in development, is termed the amplifier. These modulators selectively promote cellular immature CFTR protein production, supplying correctors and potentiator with sufficient substrate (112). For instance, patients with CF and F508del mutations received gentamicin nasal drops for 14 days, which led to a $22 \%$ increase in their wild-type CFTR function (113). Additionally, curcumin was used to treat CF by potentiating the activation of CFTR (114). Currently, triple combination therapy (elexacaftor, tezacaftor and ivacaftor) outperformed dual combination therapy (elexacaftor and tezacaftor) as it promoted the $\mathrm{Cl}^{-}$and fluid transportation, thereby further increasing the beat frequency of cilia, as manifested by in vitro efficacy in F508Del/F508Del human bronchial epithelial cells (115).

\section{Challenges and perspectives}

Despite the fact that considerable data have been obtained in regard to the molecular mechanisms of $\mathrm{CF}$, challenges still remain. Further research is required concerning the following aspects: i) Although 3-base-pair deletion and >100 related variants have been reported to account for $\mathrm{CF}$ pathogenesis, phenotypes of other variants, particularly those with single amino acid alterations, remain to be elucidated (41); ii) interpretation regarding molecular and genetic results of CFTR (whether specific variation should be defined as 'disease-prone' or 'neutral') has remained controversial, mainly due to the one-to-many association between CF genotypes to phenotypes (116), which result in difficulties in associating genetic information with clinical traits; iii) while gene therapies (gene editing) exhibit potential in CF treatment, the efficiency is decreased by high off-target effects (117); iv) another defect due to technical restriction is that prior to being intracellularly de-packaged, the transferred gene can be severely damaged by multiple natural barriers, including mucus and the immune response (118); and v) the spectrum of treatable mutations should be extended (119).

\section{Conclusions}

The current review summarizes the advances in the understanding of the molecular mechanisms underlying $\mathrm{CF}$, the corresponding molecular regulators and their clinical implementations. Emerging technology, including NGS analysis and gene therapy, will improve the understanding of the underlying molecular mechanisms. Increasing numbers of novel molecular regulators, such as miRNAs and lncRNAs, have been reported, some of which displayed potential to be biomarkers of CF. CF diagnosis was improved by carrier screening, while newborn screening facilitates the prognostic outcome via the timely intervention of CF at the early stage. The developed understanding of molecular variants (genotypes) of CF defects have enabled the development of increasingly precise and customized CF treatments, which significantly prolonged the survival of patients with $\mathrm{CF}$ with novel therapies, including gene, supplementation and modulator therapies. These have demonstrated promising future for $\mathrm{CF}$ treatment. Although rapid progresses have been reported in the understanding and treatment of $\mathrm{CF}$, improvements are required and challenges remain to be overcome.

\section{Acknowledgements}

Not applicable.

\section{Funding}

This work was supported by the National Natural Science Foundation of China (grant no. 81670004), the Guangxi Natural Science Fund Project (grant nos. 2017GXNSFAA198163, 2017GXNSFAA198149 and 2020GXNSFAA159099), the Nanning Scientific Research and Technology Development Project (grant nos. 20153124, 20163138 and 20153011), the Youth Science Foundation of Guangxi Medical University (grant no. GXMUYSF201307), the Scientific Research Project of Health Committee of Guangxi (grant nos. Z2014456 and Z2015197), the Guangxi Key Laboratory of Bio-targeting of Theranostics Fund (grant no. GXSWBX2020001), the Key R\&D plan of Qingxiu District Science and Technology Planning Project (grant no. 2020025) and the Academic Promotion Programme of Shandong First Medical University (grant no. 2019QL013).

\section{Availability of data and materials}

Not applicable.

\section{Authors' contributions}

TW, HS and YS wrote and revised the manuscript. WC, YL, ZH and QJ contributed in drafting the manuscript. CX designed the work. All authors read and approved the final manuscript.

\section{Ethics approval and consent to participate}

Not applicable.

\section{Patient consent for publication}

Not applicable. 


\section{Competing interests}

The authors declare that they have no competing interests.

\section{References}

1. Klimova B, Kuca K, Novotny M and Maresova P: Cystic fibrosis revisited-a review study. Med Chem 13: 102-109, 2017.

2. Brewington JJ, Filbrandt ET, LaRosa FJ III, Ostmann AJ, Strecker LM, Szczesniak RD and Clancy JP: Detection of CFTR function and modulation in primary human nasal cell spheroids. J Cyst Fibros 17: 26-33, 2018.

3. Keiser NW, Birket SE, Evans IA, Tyler SR, Crooke AK, Sun X, Zhou W, Nellis JR, Stroebele EK, Chu KK, et al: Defective innate immunity and hyperinflammation in newborn cystic fibrosis transmembrane conductance regulator-knockout ferret lungs. Am J Respir Cell Mol Biol 52: 683-694, 2015.

4. Santoro D, Postorino A, Lucanto C, Costa S, Cristadoro S, Pellegrino S, Conti G, Buemi M, Magazzù G and Bellinghieri G: Cystic fibrosis: A risk condition for renal disease. J Ren Nutr 27: 470-473, 2017.

5. Dekkers JF, Wiegerinck CL, de Jonge HR, Bronsveld I, Janssens HM, de Winter-de Groot KM, Brandsma AM de Jong NW, Bijvelds MJ, Scholte BJ, et al: A functional CFTR assay using primary cystic fibrosis intestinal organoids. Nat Med 19: 939-945, 2013

6. Harutyunyan M, Huang Y, Mun KS, Yang F, Arora K and Naren AP: Personalized medicine in CF: From modulator development to therapy for cystic fibrosis patients with rare CFTR mutations. Am J Physiol Lung Cell Mol Physiol 314: L529-L543, 2018.

7. Pankow S, Bamberger C, Calzolari D, Martínez-Bartolomé S, Lavallée-Adam M, Balch WE and Yates JR III: $\triangle$ F508 CFTR interactome remodelling promotes rescue of cystic fibrosis. Nature $528:$ 510-516, 2015.

8. Gadsby DC, Vergani P and Csanady L: The ABC protein turned chloride channel whose failure causes cystic fibrosis. Nature 440: 477-483, 2006

9. Rommens JM, Iannuzzi MC, Kerem B, Drumm ML, Melmer G, Dean M, Rozmahel R, Cole JL, Kennedy D, Hidaka N, et al: Identification of the cystic fibrosis gene: Chromosome walking and jumping. Science 245: 1059-1065, 1989.

10. Saint-Criq V and Gray MA: Role of CFTR in epithelial physiology. Cell Mol Life Sci 74: 93-115, 2017.

11. Cuthbert AW: New horizons in the treatment of cystic fibrosis. Br J Pharmacol 163: 173-183, 2011.

12. Zhang Z and Chen J: Atomic structure of the cystic fibrosis transmembrane conductance regulator. Cell 167: 1586-1597.e9, 2016.

13. Xue X, Mutyam V, Thakerar A, Mobley J, Bridges RJ, Rowe SM Keeling KM and Bedwell DM: Identification of the amino acids inserted during suppression of CFTR nonsense mutations and determination of their functional consequences. Hum Mol Genet 26: 3116-3129, 2017.

14. Dodge JA: A millennial view of cystic fibrosis. Dev Period Med 19: 9-13, 2015

15. Singh M, Rebordosa C, Bernholz J and Sharma N: Epidemiology and genetics of cystic fibrosis in Asia: In preparation for the next-generation treatments. Respirology 20: 1172-1181, 2015.

16. Alibakhshi R and Zamani M: Mutation analysis of CFTR gene in 70 Iranian cystic fibrosis patients. Iran J Allergy Asthma Immunol 5: 3-8, 2006.

17. Zeitlin PL: Cystic fibrosis and estrogens: A perfect storm. J Clin Invest 118: 3841-3844, 2008.

18. Lui JK, Kilch J, Fridlyand S, Dheyab A and Bielick Kotkowski C: Non-classic cystic fibrosis: The value in family history. Am J Med 130: e333-e334, 2017.

19. Thomas JM, Durack A, Sterling A, Todd PM and Tomson N: Aquagenic wrinkling of the palms: A diagnostic clue to cystic fibrosis carrier status and non-classic disease. Lancet 389: 846 , 2017.

20. Severiche-Bueno D, Gamboa E, Reyes LF and Chotirmall SH: Hot topics and current controversies in non-cystic fibrosis bronchiectasis. Breathe (Sheff) 15: 286-295, 2019.

21. Andersen DH: Cystic fibrosis of the pancreas and its relation to celiac disease. Am J Dis Child 56, 1938.

22. Bell SC, Mall MA, Gutierrez H, Macek M, Madge S, Davies JC, Burgel PR, Tullis E, Castaños C, Castellani C, et al: The future of cystic fibrosis care: A global perspective. Lancet Respir Med 8: $65-124,2020$.
23. Gibson LE and Cooke RE: A test for concentration of electrolytes in sweat in cystic fibrosis of the pancreas utilizing pilocarpine by iontophoresis. Pediatrics 23: 545-549, 1959.

24. Di Sant'agnese PA, Darling RC, Perera GA and Shea E: Abnormal electrolyte composition of sweat in cystic fibrosis of the pancreas; clinical significance and relationship to the disease. Pediatrics 12: 549-563, 1953.

25. Davis PB: Cystic fibrosis since 1938. Am J Respir Crit Care Med 173: 475-482, 2006.

26. Yamada A, Komaki Y, Komaki F, Micic D, Zullow S and Sakuraba A: Risk of gastrointestinal cancers in patients with cystic fibrosis: A systematic review and meta-analysis. Lancet Oncol 19: 758-767, 2018.

27. Goetz D and Ren CL: Review of cystic fibrosis. Pediatr Ann 48: e154-e161, 2019.

28. Fanen P, Wohlhuter-Haddad A and Hinzpeter A: Genetics of cystic fibrosis: CFTR mutation classifications toward genotype-based CF therapies. Int J Biochem Cell Biol 52: 94-102, 2014.

29. Linsdell P: Cystic fibrosis transmembrane conductance regulator (CFTR): Making an ion channel out of an active transporter structure. Channels (Austin) 12: 284-290, 2018.

30. Moran O: The gating of the CFTR channel. Cell Mol Life Sci 74: 85-92, 2017.

31. Mall MA and Galietta LJ: Targeting ion channels in cystic fibrosis. J Cyst Fibros 14: 561-570, 2015

32. Gentzsch M and Mall MA: Ion channel modulators in cystic fibrosis. Chest 154: 383-393, 2018.

33. Shah VS, Meyerholz DK, Tang XX, Reznikov L, Abou Alaiwa M, Ernst SE, Karp PH, Wohlford-Lenane CL, Heilmann KP, Leidinger MR, et al: Airway acidification initiates host defense abnormalities in cystic fibrosis mice. Science 351: 503-507, 2016.

34. Liu F, Zhang Z, Csanády L, Gadsby DC and Chen J: Molecular structure of the human CFTR ion channel. Cell 169: 85-95.e8, 2017.

35. Cook DP, Rector MV, Bouzek DC, Michalski AS, Gansemer ND, Reznikov LR, Li X, Stroik MR, Ostedgaard LS, Abou Alaiwa MH, et al: Cystic fibrosis transmembrane conductance regulator in sarcoplasmic reticulum of airway smooth muscle. Implications for airway contractility. Am J Respir Crit Care Med 193: 417-426, 2016.

36. Iitiä A, Høgdall E, Dahlen P, Hurskainen P, Vuust J and Siitari H: Detection of mutation delta F508 in the cystic fibrosis gene using allele-specific PCR primers and time-resolved fluorometry. PCR Methods Appl 2: 157-162, 1992.

37. Xia E, Zhang Y, Cao H, Li J, Duan R and Hu J: TALEN-mediated gene targeting for cystic fibrosis-gene therapy. Genes (Basel) 10: 39, 2019.

38. Costa C, Pruliere-Escabasse V, de Becdelievre A, Gameiro C, Golmard L, Guittard C, Bassinet L, Bienvenu T, Georges MD, Epaud R, et al: A recurrent deep-intronic splicing CF mutation emphasizes the importance of mRNA studies in clinical practice. J Cyst Fibros 10: 479-482, 2011.

39. Brandt C, Roehmel J, Rickerts V, Melichar V, Niemann N and Schwarz C: Aspergillus bronchitis in patients with cystic fibrosis. Mycopathologia 183: 61-69, 2018.

40. Strom CM, Ginsberg N, Rechitsky S, Cieslak J, Ivakhenko V, Wolf G, Lifchez A, Moise J, Valle J, Kaplan B, et al: Three births after preimplantation genetic diagnosis for cystic fibrosis with sequential first and second polar body analysis. Am J Obstet Gynecol 178: 1298-1306, 1998.

41. Girardet A, Viart V, Plaza S, Daina G, De Rycke M, Des Georges M, Fiorentino F, Harton G, Ishmukhametova A, Navarro J, et al: The improvement of the best practice guidelines for preimplantation genetic diagnosis of cystic fibrosis: Toward an international consensus. Eur J Hum Genet 24: 469-478, 2016.

42. Brennan ML and Schrijver I: Cystic fibrosis: A review of associated phenotypes, use of molecular diagnostic approaches, genetic characteristics, progress, and dilemmas. J Mol Diagn 18: 3-14, 2016.

43. Bell CJ, Dinwiddie DL, Miller NA, Hateley SL, Ganusova EE, Mudge J, Langley RJ, Zhang L, Lee CC, Schilkey FD, et al: Carrier testing for severe childhood recessive diseases by next-generation sequencing. Sci Transl Med 3: 65ra4, 2011.

44. Rengaraju B, Thana K, La A, Pavithra K, Durairaj V, Challapalli SH and Das A: Inquest of the SNP in cystic fibrosis-A bioinformatic approach. Int J Curr Microbiol Appl Sci 6: 1255-1263, 2017

45. Beauchamp KA, Johansen Taber KA, Grauman PV, Spurka L, Lim-Harashima J, Svenson A, Goldberg JD and Muzzey D: Sequencing as a first-line methodology for cystic fibrosis carrier screening. Genet Med 21: 2569-2576, 2019. 
46. Baker MW, Atkins AE, Cordovado SK, Hendrix M, Earley MC and Farrell PM: Improving newborn screening for cystic fibrosis using next-generation sequencing technology: A technical feasibility study. Genet Med 18: 231-238, 2016.

47. Marangi $M$ and Pistritto G: Innovative therapeutic strategies for cystic fibrosis: Moving forward to CRISPR technique. Front Pharmacol 9: 396, 2018.

48. Hodges CA and Conlon RA: Delivering on the promise of gene editing for cystic fibrosis. Genes Dis 6: 97-108, 2019.

49. Park S and Beal PA: Off-target editing by CRISPR-guided DNA base editors. Biochemistry 58: 3727-3734, 2019.

50. Schwank G, Koo BK, Sasselli V, Dekkers JF, Heo I, Demircan T, Sasaki N, Boymans S, Cuppen E, van der Ent CK, et al: Functional repair of CFTR by CRISPR/Cas9 in intestinal stem cell organoids of cystic fibrosis patients. Cell Stem Cell 13: 653-658, 2013

51. Crane AM, Kramer P, Bui JH, Chung WJ, Li XS Gonzalez-Garay ML, Hawkins F, Liao W, Mora D, Choi S, et al: Targeted correction and restored function of the CFTR gene in cystic fibrosis induced pluripotent stem cells. Stem Cell Reports 4: 569-577, 2015 .

52. Liang $\mathrm{P}$, Xu Y, Zhang X, Ding C, Huang R, Zhang Z, Lv J, Xie X, Chen Y, Li Y, et al: CRISPR/Cas9-mediated gene editing in human tripronuclear zygotes. Protein Cell 6: 363-372, 2015

53. Saayman SM, Ackley A, Burdach J, Clemson M, Gruenert DC, Tachikawa K, Chivukula P, Weinberg MS and Morris KV: Long non-coding RNA BGas regulates the cystic fibrosis transmembrane conductance regulator. Mol Ther 24: 1351-1357, 2016.

54. Dimartino D, Colantoni A, Ballarino M, Martone J, Mariani D, Danner J, Bruckmann A, Meister G, Morlando M and Bozzoni I: The long non-coding RNA lnc-31 interacts with Rock1 mRNA and mediates its YB-1-dependent translation. Cell Rep 23: 733-740, 2018

55. Kishore $\mathrm{S}$ and Stamm S: The snoRNA HBII-52 regulates alternative splicing of the serotonin receptor 2C. Science 311: 230-232, 2006.

56. Gil N and Ulitsky I: Regulation of gene expression by cis-acting long non-coding RNAs. Nat Rev Genet 21: 102-117, 2020.

57. Fabbri E, Tamanini A, Jakova T, Gasparello J, Manicardi A, Corradini R, Sabbioni G, Finotti A, Borgatti M, Lampronti I, et al: A peptide nucleic acid against MicroRNA miR-145-5p enhances the expression of the cystic fibrosis transmembrane conductance regulator (CFTR) in Calu-3 cells. Molecules 23: 71, 2017.

58. Megiorni F, Cialfi S, Dominici C, Quattrucci S and Pizzuti A: Synergistic post-transcriptional regulation of the Cystic Fibrosis Transmembrane conductance Regulator (CFTR) by miR-101 and miR-494 specific binding. PLoS One 6: e26601, 2011.

59. Li Z, Yao JN, Huang WT, He RQ, Ma J, Chen G and Wei QJ: Expression of miR-542-3p in osteosarcoma with miRNA microarray data, and its potential signaling pathways. Mol Med Rep 19: 974-983, 2019.

60. Hassan F, Nuovo GJ, Crawford M, Boyaka PN, Kirkby S, Nana-Sinkam SP and Cormet-Boyaka E: MiR-101 and miR-144 regulate the expression of the CFTR chloride channel in the lung. PLoS One 7: e50837, 2012

61. Ramachandran S, Karp PH, Jiang P, Ostedgaard LS, Walz AE, Fisher JT, Keshavjee S, Lennox KA, Jacobi AM, Rose SD, et al: A microRNA network regulates expression and biosynthesis of wild-type and DeltaF508 mutant cystic fibrosis transmembrane conductance regulator. Proc Natl Acad Sci USA 109 13362-13367, 2012.

62. Fesen K, Silveyra P, Fuentes N, Nicoleau M, Rivera L, Kitch D, Graff GR and Siddaiah R: The role of microRNAs in chronic pseudomonas lung infection in Cystic fibrosis. Respir Med 151: 133-138, 2019.

63. Balloy V, Koshy R, Perra L, Corvol H, Chignard M, Guillot L and Scaria V: Bronchial epithelial cells from cystic fibrosis patients express a specific long non-coding RNA signature upon Pseudomonas aeruginosa infection. Front Cell Infect Microbiol 7: 218, 2017.

64. McKiernan PJ, Molloy K, Cryan SA, McElvaney NG and Greene CM: Long noncoding RNA are aberrantly expressed in vivo in the cystic fibrosis bronchial epithelium. Int $\mathrm{J}$ Biochem Cell Biol 52: 184-191, 2014.

65. Kumar P, Sen C, Peters K, Frizzell RA and Biswas R: Comparative analyses of long non-coding RNA profiles in vivo in cystic fibrosis lung airway and parenchyma tissues. Respir Res 20: 284, 2019.

66. McKiernan PJ, Cunningham O, Greene CM and Cryan SA: Targeting miRNA-based medicines to cystic fibrosis airway epithelial cells using nanotechnology. Int J Nanomed 8: 3907-3915, 2013.
67. Lu Q, Liu T, Feng H, Yang R, Zhao X, Chen W, Jiang B, Qin H, Guo X, Liu M, et al: Circular RNA circSLC8A1 acts as a sponge of $\mathrm{miR}-130 \mathrm{~b} / \mathrm{miR}-494$ in suppressing bladder cancer progression via regulating PTEN. Mol Cancer 18: 111, 2019.

68. Yu CY, Li TC, Wu YY, Yeh CH, Chiang W, Chuang CY and Kuo HC: The circular RNA circBIRC6 participates in the molecular circuitry controlling human pluripotency. Nat Commun 8: 1149, 2017.

69. Nowacka-Zawisza M and Wiśnik E: DNA methylation and histone modifications as epigenetic regulation in prostate cancer (Review). Oncol Rep 38: 2587-2596, 2017.

70. Sirinupong $\mathrm{N}$ and Yang Z: Epigenetics in cystic fibrosis: Epigenetic targeting of a genetic disease. Curr Drug Targets 16: 976-987, 2015.

71. Morandini AC, Santos CF and Yilmaz Ö: Role of epigenetics in modulation of immune response at the junction of host-pathogen interaction and danger molecule signaling. Pathog Dis 74: ftw082, 2016.

72. Chen Y, Armstrong DA, Salas LA, Hazlett HF, Nymon AB, Dessaint JA, Aridgides DS, Mellinger DL, Liu X, Christensen BC and Ashare A: Genome-wide DNA methylation profiling shows a distinct epigenetic signature associated with lung macrophages in cystic fibrosis. Clin Epigenetics 10: 152, 2018

73. Magalhães M, Tost J, Pineau F, Rivals I, Busato F, Alary N, Mely L, Leroy S, Murris M, Caimmi D, et al: Dynamic changes of DNA methylation and lung disease in cystic fibrosis: Lessons from a monogenic disease. Epigenomics 10: 1131-1145, 2018.

74. Scott M and De Sario A: DNA methylation changes in cystic fibrosis: Cause or consequence? Clin Genet 98: 3-9, 2020.

75. Hutt DM, Herman D, Rodrigues AP, Noel S, Pilewski JM, Matteson J, Hoch B, Kellner W, Kelly JW, Schmidt A, et al: Reduced histone deacetylase 7 activity restores function to misfolded CFTR in cystic fibrosis. Nat Chem Biol 6: 25-33, 2010.

76. Bartling TR and Drumm ML: Loss of CFTR results in reduction of histone deacetylase 2 in airway epithelial cells. Am J Physiol Lung Cell Mol Physiol 297: L35-L43, 2009.

77. Rymut SM, Harker A, Corey DA, Burgess JD, Sun H, Clancy JP and Kelley TJ: Reduced microtubule acetylation in cystic fibrosis epithelial cells. Am J Physiol Lung Cell Mol Physiol 305: L419-L431, 2013.

78. Bergougnoux A, Rivals I, Liquori A, Raynal C, Varilh J, Magalhães M, Perez MJ, Bigi N, Des Georges M, Chiron R, et al: A balance between activating and repressive histone modifications regulates cystic fibrosis transmembrane conductance regulator (CFTR) expression in vivo. Epigenetics 9: 1007-1017, 2014.

79. Cutting GR: Cystic fibrosis genetics: From molecular understanding to clinical application. Nat Rev Genet 16: 45-56, 2015.

80. Davis PB, Drumm M and Konstan MW: Cystic fibrosis. Am J Respir Crit Care Med 154: 1229-1256, 1996.

81. De Boeck K, Vermeulen F and Dupont L: The diagnosis of cystic fibrosis. Presse Med 46: e97-e108, 2017.

82. Schwarzenberg SJ, Hempstead SE, McDonald CM, Powers SW, Wooldridge J, Blair S, Freedman S, Harrington E, Murphy PJ, Palmer L, et al: Enteral tube feeding for individuals with cystic fibrosis: Cystic Fibrosis Foundation evidence-informed guidelines. J Cyst Fibros 15: 724-735, 2016.

83. Guglani L, Moir D and Jain A: Sweat chloride concentrations in children with Idiopathic Nephrotic Syndrome. Pediatr Pulmonol 51: 49-52, 2016.

84. Brown A, Jenkins L, Reid A, Leavy A, McDowell G, McIlroy C, Thompson A and McNaughten B: How to perform and interpret the sweat test. Arch Dis Child Educ Pract Ed 105: 230-235, 2019

85. Solomon GM, Liu B, Sermet-Gaudelus I, Fajac I, Wilschanski M, Vermeulen F and Rowe SM: A multiple reader scoring system for Nasal Potential Difference parameters. J Cyst Fibros 16: 573-578, 2017.

86. Solomon GM, Bronsveld I, Hayes K, Wilschanski M, Melotti P, Rowe SM and Sermet-Gaudelus I: Standardized measurement of nasal membrane transepithelial potential difference (NPD). J Vis Exp: 57006, 2018.

87. Beka $\mathrm{M}$ and Leal T: Nasal potential difference to quantify trans-epithelial ion transport in mice. J Vis Exp: 57934, 2018.

88. Old RW, Bestwick JP and Wald NJ: Prenatal maternal plasma DNA screening for cystic fibrosis: A computer modelling study of screening performance. F1000Res 6: 1896, 2017.

89. Sugunaraj JP, Brosius HM, Murray MF, Manickam K, Stamm JA, Carey DJ and Mirshahi UL: Predictive value of genomic screening: Cross-sectional study of cystic fibrosis in 50,788 electronic health records. NPJ Genom Med 4: 21, 2019. 
90. Ferlin A and Stuppia L: Diagnostics of CFTR-negative patients with congenital bilateral absence of vas deferens: Which mutations are of most interest? Expert Rev Mol Diagn 20: 265-267, 2020

91. Wagener JS, Sontag MK and Accurso FJ: Newborn screening for cystic fibrosis. Curr Opin Pediatr 15: 309-315, 2003.

92. O'Brien TJ and Welch M: Recapitulation of polymicrobial communities associated with cystic fibrosis airway infections: A perspective. Future Microbiol 14: 1437-1450, 2019.

93. Lyczak JB, Cannon CL and Pier GB: Lung infections associated with cystic fibrosis. Clin Microbiol Rev 15: 194-222, 2002.

94. Savant AP and McColley SA: Cystic fibrosis year in review 2016. Pediatr Pulmonol 52: 1092-1102, 2017.

95. Wilson J: Treating genes and patients. Gene Ther 27: 109-110, 2020.

96. Rafeeq MM and Murad HAS: Cystic fibrosis: Current therapeutic targets and future approaches. J Transl Med 15: 84, 2017.

97. Moss RB, Flume PA, Elborn JS, Cooke J, Rowe SM, McColley SA, Rubenstein RC and Higgins M; VX11-770-110 (KONDUCT) Study Group: Efficacy and safety of ivacaftor in patients with cystic fibrosis who have an Arg117His-CFTR mutation: A double-blind, randomised controlled trial. Lancet Respir Med 3: 524-533, 2015.

98. Arjmand B, Larijani B, Sheikh Hosseini M, Payab M, Gilany K, Goodarzi P, Parhizkar Roudsari P, Amanollahi Baharvand M and Hoseini Mohammadi NS: The horizon of gene therapy in modern medicine: Advances and challenges. Adv Exp Med Biol 1247: 33-64, 2020.

99. Yang Q, Soltis AR, Sukumar G, Zhang X, Caohuy H, Freedy J, Dalgard CL, Wilkerson MD, Pollard HB and Pollard BS: Gene therapy-emulating small molecule treatments in cystic fibrosis airway epithelial cells and patients. Respir Res 20: 290, 2019.

100.Eymery M, Morfin F, Doleans-Jordheim A, Perceval M, Ohlmann C, Mainguy C and Reix P: Viral respiratory tract infections in young children with cystic fibrosis: A prospective full-year seasonal study. Virol J 16: 111, 2019.

101. Tümmler B: Treatment of cystic fibrosis with CFTR modulators Pneumologie 70: 301-313, 2016 (In German).

102. Bessonova L, Volkova N, Higgins M, Bengtsson L, Tian S, Simard C, Konstan MW, Sawicki GS, Sewall A, Nyangoma $S$, et al: Data from the US and UK cystic fibrosis registries support disease modification by CFTR modulation with ivacaftor. Thorax 73: 731-740, 2018.

103. Faruqi S, Shiferaw D and Morice AH: Effect of ivacaftor on objective and subjective measures of cough in patients with cystic fibrosis. Open Respir Med J 10: 105-108, 2016.

104. Heltshe SL, Mayer-Hamblett N, Burns JL, Khan U, Baines A, Ramsey BW and Rowe SM; GOAL (the G551D Observation-AL) Investigators of the Cystic Fibrosis Foundation Therapeutics Development Network: Pseudomonas aeruginosa in cystic fibrosis patients with G551D-CFTR treated with ivacaftor. Clin Infect Dis 60: 703-712, 2015.

105. Krainer G, Schenkel M, Hartmann A, Ravamehr-Lake D, Deber CM and Schlierf M: CFTR transmembrane segments are impaired in their conformational adaptability by a pathogenic loop mutation and dynamically stabilized by Lumacaftor. J Biol Chem 295: 1985-1991, 2020.

106. Wainwright CE,ElbornJS and Ramsey BW:Lumacaftor-ivacaftor in patients with cystic fibrosis homozygous for Phe508del CFTR. N Engl J Med 373: 1783-1784, 2015.
107. Konstan MW, McKone EF, Moss RB, Marigowda G, Tian S, Waltz D, Huang X, Lubarsky B, Rubin J, Millar SJ, et al: Assessment of safety and efficacy of long-term treatment with combination lumacaftor and ivacaftor therapy in patients with cystic fibrosis homozygous for the F508del-CFTR mutation (PROGRESS): A phase 3, extension study. Lancet Respir Med 5: 107-118, 2017.

108. Sala MA and Jain M: Tezacaftor for the treatment of cystic fibrosis. Expert Rev Respir Med 12: 725-732, 2018.

109. Rowe SM, Daines C, Ringshausen FC, Kerem E, Wilson J, Tullis E, Nair N, Simard C, Han L, Ingenito EP, et al: Tezacaftor-ivacaftor in residual-function heterozygotes with cystic fibrosis. N Engl J Med 377: 2024-2035, 2017.

110. Donaldson SH, Pilewski JM, Griese M, Cooke J, Viswanathan L, Tullis E, Davies JC, Lekstrom-Himes JA and Wang LT; VX11-661-101 Study Group: Tezacaftor/ivacaftor in subjects with cystic fibrosis and F508del/F508del-CFTR or F508del/G551D-CFTR. Am J Respir Crit Care Med 197: 214-224, 2018

111. Taylor-Cousar JL, Munck A, McKone EF, van der Ent CK, Moeller A, Simard C, Wang LT, Ingenito EP, McKee C, $\mathrm{Lu}$ Y, et al: Tezacaftor-Ivacaftor in Patients with Cystic Fibrosis Homozygous for Phe508del. N Engl J Med 377: 2013-2023, 2017.

112. Giuliano KA, Wachi S, Drew L, Dukovski D, Green O, Bastos C, Cullen MD, Hauck S, Tait BD, Munoz B, et al: Use of a high-throughput phenotypic screening strategy to identify amplifiers, a novel pharmacological class of small molecules that exhibit functional synergy with potentiators and correctors. SLAS Discov 23: 111-121, 2018.

113. Gambari R, Breveglieri G, Salvatori F, Finotti A and Borgatti M: Therapy for cystic fibrosis caused by nonsense mutations. Cystic Fibrosis in the Light of New Research Ch. 13, 2015.

114. Wang G: Interplay between inhibitory ferric and stimulatory curcumin regulates phosphorylation-dependent human cystic fibrosis transmembrane conductance regulator and DeltaF508 activity. Biochemistry 54: 1558-1566, 2015.

115. Chaudary N: Triplet CFTR modulators: Future prospects for treatment of cystic fibrosis. Ther Clin Risk Manag 14: 2375-2383, 2018.

116. Raynal C, Baux D, Theze C, Bareil C, Taulan M, Roux AF, Claustres M, Tuffery-Giraud S and des Georges M: A classification model relative to splicing for variants of unknown clinical significance: Application to the CFTR gene. Hum Mutat 34: 774-784, 2013.

117. Mention K, Santos L and Harrison PT: Gene and base editing as a therapeutic option for cystic fibrosis-learning from other diseases. Genes (Basel) 10: 387, 2019.

118. Osman G, Rodriguez J, Chan SY, Chisholm J, Duncan G, Kim N, Tatler AL, Shakesheff KM, Hanes J, Suk JS and Dixon JE: PEGylated enhanced cell penetrating peptide nanoparticles for lung gene therapy. J Control Release 285: 35-45, 2018.

119. Condren ME and Bradshaw MD: Ivacaftor: A novel gene-based therapeutic approach for cystic fibrosis. J Pediatr Pharmacol Ther 18: 8-13, 2013.

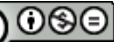

This work is licensed under a Creative Commons Attribution-NonCommercial-NoDerivatives 4.0 International (CC BY-NC-ND 4.0) License. 